\title{
Fuzzy Gains-Scheduling of an Integral Sliding Mode Controller for a Quadrotor Unmanned Aerial Vehicle
}

\author{
Nour Ben Ammar, Soufiene Bouallègue, Joseph Haggège \\ Research Laboratory in Automatic Control (LA.R.A.), \\ National Engineering School of Tunis (ENIT), \\ University of Tunis El Manar, \\ BP 37, Le Belvédère, \\ 1002 Tunis, Tunisia
}

\begin{abstract}
This paper investigates an Adaptive Fuzzy GainsScheduling Integral Sliding Mode Controller (AFGS-ISMC) design approach to deal with the attitude and altitude stabilization problem of an Unmanned Aerial Vehicles (UAV) precisely of a quadrotor. The Integral Sliding Mode Control (ISMC) seems to be an adequate control tool to remedy this problem. The selection of the controller parameters is done most of the time using repetitive trials-errors based methods. This method is not completely reliable and becomes a time-consuming and difficult task. Here we propose the tuning and selection of all ISMC gains adaptively according to a fuzzy supervisor. The sliding surface and its differential are declared as Fuzzy Logic Supervisor (FLS) inputs and the integral sliding mode control gains as the FLS outputs. The proposed fuzzy-based supervision mechanisms modify all ISMC gains to be time-varying and further enhance the performance and robustness of the obtained adaptive nonlinear controllers against uncertainties and external disturbances. The proposed adaptive fuzzy technique increases the effectiveness of the ISMC structure compared to the classical SMC strategy and excludes the dull and repetitive trials-errors process for its design and tuning. Various simulations have been carried out and followed by comparison and discussion of the results in order to prove the superiority of the suggested fuzzy gains-scheduled ISMC approach for the quadrotor attitude and altitude flight stabilization.
\end{abstract}

Keywords-Quadrotor UAV; modeling; flight dynamics stabilization; integral sliding mode control; fuzzy gains-scheduling, adaptive control

\section{INTRODUCTION}

Unmanned Aerial Vehicles (UAVs) are volant robots with no aviator that are capable of carrying out various missions in inimical and unsettled environments [1]. The quadrotor, a type of these UAVs, is a very promising concept with a Vertical Take-Off and Landing (VTOL) motion thanks to four rotors which are independently controlled [2]-[7]. These rotorcrafts have been developed to perform various tasks in different fields whether in the military or even civilian. As a class of unmanned rotorcraft, quadrotors are arising as an incomparable and promising stand for various tasks such as recognition, surveillance, environmental monitoring, life-saving operations and aerial photography through their VTOL capacity yet its structure is simple. In some aspects, the quadrotors have better maneuverability than other VTOL vehicles due to the four rotors which can increase the mobility and load ability.
Unfortunately, the difficulty of control design of such a type of rotorcrafts increases under the dynamics nonlinearity, parametric uncertainties and external disturbances. Moreover, the dynamical model of a quadrotor UAV has six Degree-Of Freedom (DOF) with only four independent thrust forces generated by four rotors. It is difficult to control all these six outputs with only four control inputs. For this problem, it is necessary to use adequate control methods such as the nonlinear ones to design robust and effective flight controllers. Nonlinear control is one of the significant challenges in the modern control theory [8], [9]. Facing this defiance, it is obvious that there is not a particular procedure that must be applied to all nonlinear systems. So, we must resort to employing the best adapted tools to the current problem. In this context, the Sliding Mode Control (SMC) strategy presents a promising solution [10]-[15].

The SMC approach is a control technique known for its robustness for the complex and nonlinear systems. The best constructive characteristic of this controller is in the total adjustment of the perturbation wherever the system is in the sliding phase and a sliding mode is imposed. This last one takes place when the state is on an appropriate subspace of the state-space. The compensated dynamics become insensible to perturbation and uncertainties below the SMC design [16][18]. Sliding mode control has been successfully applied to robot manipulators, high-performance electric motors, underwater vehicles and UAV [19]. Regrettably, a perfect sliding mode controller has a discontinuous switching function which causes a fast switching of the signal from one value to another. Due to the limitation of physics and the finite time delay of the control computation, it is intolerable to attain boundedly fast switching control in the practical implementation [20], [21].

In the literature, the Integral Sliding Mode Control (ISMC) variant appears at first as an answer to the achieving phase question for systems with matched disturbances only [23]. Recently, the ISMC technique has been used in order to analyze the problem of minimizing the disturbance of systems taken into account a nonlinear drift term and a constant input matrix [3], [19]. This outcome has been applied evenly in connection with different control strategies like the Model Predictive Control (MPC) in [25]. In [26] an integral sliding mode altitude control for a small model helicopter with ground effect compensation is proposed. The authors then present the 
implementation of an integral action on the controller based on sliding modes for a conventional helicopter. In [27], an adaptive integral sliding mode control for a small scale quadrotor is designed to online estimate the attitude controllers' parameters. Authors in [28] applied the L1 adaptive control theory to design the attitude stabilization against the model uncertainties and environmental disturbances.

Based on the aforementioned studies, the main challenging stage in the ISMC design for quadrotors UAV is the choice of appropriate controllers' gains that define, as the effective control parameters, the dynamics of such feedback controllers. Such gains tuning provides a desired balance between the state variable responses and control efforts. In the ISMC framework, these decision variables are selected by repetitive trials-errors based methods that become time consuming and difficult task [4], [5]. Indeed, the methods described above in [22]-[25] are interesting but may not lead to satisfactory results because they are usually time-consuming and very restrictive. Looking for new ways to handle these complex problems, a systematic approach to tune these design parameters is then an interesting task in the sliding mode control of VTOL rotorcrafts.

Incited by its noticeable draw in diverse control appliance as well as its straightforwardness in real-world implementation, the fuzzy control theory has been applied to attain advanced performances and robustness for complex and nonlinear systems [26]-[28].The tuning and selection of all ISMC gains, systematically and without any trials-errors based stage, thanks to a proposed fuzzy supervision mechanism, is a promising idea and efficient solution given the complexity and the hardness design of the conventional ISMC approach. Such a proposed fuzzy gains-scheduling technique allows having variable gains over time based integral sliding mode controllers that are more appropriate and efficient to uncertainties, disturbances and faults of UAV rotorcrafts. So, the principal contribution of this paper is to propound a novel strategy to conceit and adjust adaptive integral sliding mode controllers for the attitude and altitude stabilization problem of a quadrotor. Both gains of the sliding surfaces and sign functions selection problem is formulated and is efficiently solved thanks to proposed fuzzy supervision mechanisms.

The remained of this paper is organized as follows. In Section II, a mathematical nonlinear model of the quadrotor is presented thanks to the Euler-Newton formalism. In Section III, the adaptive fuzzy gains-scheduling integral sliding mode controller problem is formulated for the altitude and attitude quadrotors dynamics' stabilization. All sliding mode controllers' gains, as effective design parameters, are scheduled based on proposed fuzzy supervision mechanisms leading to reduce the chattering phenomenon. In Section IV, various simulations are done to point the efficacy the proposed fuzzy-based sliding mode controllers for the flight stabilization of the UAV drone. Finally, conclusions are drawn in Section V.

\section{MODELING OF THE QUADROTOR UAV}

A quadrotor is an UAV with four rotors that are controlled independently as shown in Fig. 1. The movement of the quadrotor results from changes in the speed of the rotors. The quadrotor structure is assumed to be rigid and symmetrical. The propellers are rigid and the thrust and drag forces are proportional to the square of propeller's speed [1], [2], [6], [7].

To develop a mathematical model of such device, both coordinate systems such as the earth-frame $\boldsymbol{F}_{E}=\left\{O_{e}, \boldsymbol{x}_{e}, \boldsymbol{y}_{e}, \boldsymbol{z}_{e}\right\} \quad$ and the body-frame $\boldsymbol{F}_{B}=\left\{\boldsymbol{O}_{b}, \boldsymbol{x}_{b}, \boldsymbol{y}_{b}, \boldsymbol{z}_{b}\right\}$ are considered [29]. Let denote by $m$ the total mass of the quadrotor, $g$ the acceleration of the gravity and $l$ the distance from the center of each rotor to the Center (COG) [].

The orientation of the quadrotor is given by the rotation matrix $\boldsymbol{R}: \boldsymbol{F}_{E} \rightarrow \boldsymbol{F}_{B}$ which depends on the Euler angles $(\phi, \theta, \psi)$ and defined by the following equation:

$$
\boldsymbol{R}(\phi, \theta, \psi)=\left[\begin{array}{ccc}
c \psi c \theta & s \phi s \theta c \psi-s \psi c \theta & c \phi s \theta c \psi+s \psi s \phi \\
s \psi c \theta & s \phi s \theta s \psi+c \psi c \theta & c \phi s \theta s \psi-s \phi c \psi \\
-s \theta & s \phi c \theta & c \phi c \theta
\end{array}\right]
$$

where $c()=.\cos ($.$) and s()=.\sin ($.$) .$

The position and the attitude angles of the quadrotor in the earth-frame are defined as $\xi=[x, y, z]^{T}$ and $\boldsymbol{\eta}=[\phi, \theta, \psi]^{T}, \quad$ respectively. We denote by $-\pi / 2 \leq \phi \leq \pi / 2,-\pi / 2 \leq \theta \leq \pi / 2$ and $-\pi \leq \psi \leq \pi$ the roll, pitch and yaw angles, respectively. The complete dynamical model of the studied quadrotor is established applying the Newton-Euler formalism. The Newton's laws convey to the pursuant motion equations [1]-[3]:

$$
\left\{\begin{array}{l}
m \ddot{\xi}=\boldsymbol{F}_{t h}+\boldsymbol{F}_{d}+\boldsymbol{F}_{g} \\
\boldsymbol{J} \dot{\boldsymbol{\Omega}}=\boldsymbol{M}-\boldsymbol{M}_{g p}-\boldsymbol{M}_{g b}-\boldsymbol{M}_{a}
\end{array}\right.
$$

where $\boldsymbol{F}_{t h}=\boldsymbol{R}(\phi, \theta, \psi)\left[0,0, \sum_{i=1}^{4} F_{i}\right]^{T}$ denotes the total thrust force of the four rotors, $\boldsymbol{F}_{d}=\operatorname{diag}\left(\kappa_{1}, \kappa_{2}, \kappa_{3}\right) \dot{\boldsymbol{\xi}}^{T}$ is the air drag force which resists to the quadrotor motion, $\boldsymbol{F}_{g}=[0,0, m g]^{T}$ is the gravity force, $\boldsymbol{M}=\left[\tau_{\phi}, \tau_{\theta}, \tau_{\psi}\right]^{T}$ represents the total rolling, pitching and yawing moments, $\boldsymbol{M}_{g p}$ and $\boldsymbol{M}_{g b}$ are the propellers and quadrotor body gyroscopic torques, respectively, and $\boldsymbol{M}_{a}=\operatorname{diag}\left(\kappa_{4}, \kappa_{5}, \kappa_{6}\right)\left[\dot{\phi}^{2}, \dot{\theta}^{2}, \dot{\psi}^{2}\right]^{T}$ is the moment resulting from the aerodynamic frictions [29]. 


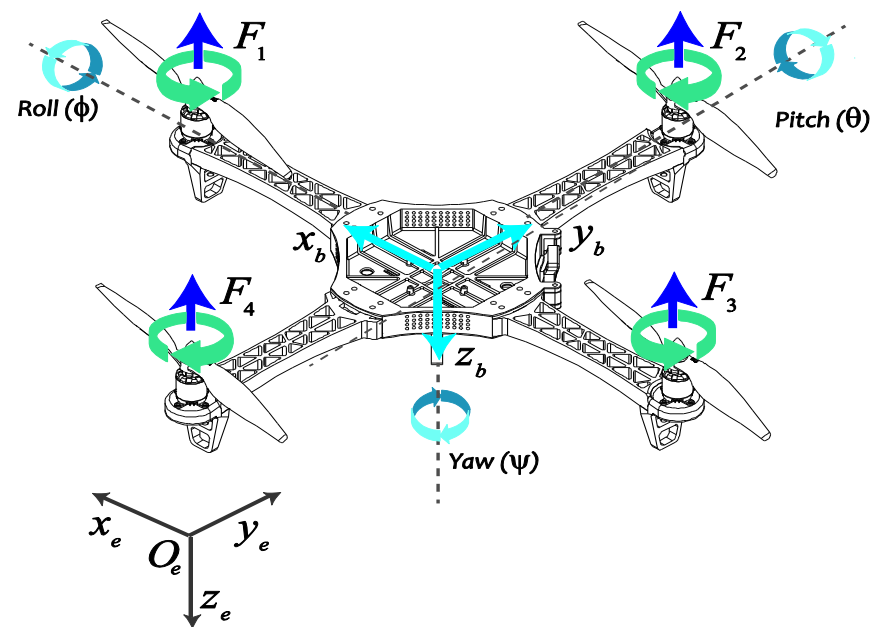

Fig. 1. Mechanical structure of the quadrotor and related frames.

By replacing the position vector and the forces expressions in (2), we acquire the next translational dynamics of the quadrotor:

$$
\left\{\begin{array}{l}
\ddot{x}=\frac{1}{m}(c \phi c \psi s \theta+s \phi s \psi) u_{1}-\frac{\kappa_{1}}{m} \dot{x} \\
\ddot{y}=\frac{1}{m}(c \phi s \psi s \theta-s \phi c \psi) u_{1}-\frac{\kappa_{2}}{m} \dot{y} \\
\ddot{z}=\frac{1}{m} c \phi c \theta u_{1}-g-\frac{\kappa_{3}}{m} \dot{z}
\end{array}\right.
$$

From the second part of (2), we deduct the rotational dynamics of the quadrotors:

$$
\left\{\begin{array}{l}
\ddot{\phi}=\frac{\left(I_{y}-I_{z}\right)}{I_{x}} \dot{\theta} \dot{\psi}-\frac{J_{r}}{I_{x}} \overline{\Omega_{r}} \dot{\theta}-\frac{\kappa_{4}}{I_{x}} \dot{\phi}^{2}+\frac{1}{I_{x}} u_{2} \\
\ddot{\theta}=\frac{\left(I_{z}-I_{x}\right)}{I_{y}} \dot{\phi} \dot{\psi}-\frac{J_{r}}{I_{y}} \bar{\Omega}_{r} \dot{\phi}-\frac{\kappa_{5}}{I_{y}} \dot{\theta}^{2}+\frac{1}{I_{y}} u_{3} \\
\ddot{\psi}=\frac{\left(I_{x}-I_{y}\right)}{I_{z}} \dot{\theta} \dot{\phi}-\frac{\kappa_{6}}{I_{z}} \dot{\psi}^{2}+\frac{1}{I_{z}} u_{4}
\end{array}\right.
$$

where $\bar{\Omega}_{r}=\omega_{1}-\omega_{2}+\omega_{3}-\omega_{4}$ denotes the overall residual rotor angular speed, $I_{x}, I_{y}$ and $I_{z}$ are the body inertia, $J_{r}$ is the rotor inertia and $\kappa_{i}, i=1,2, \ldots, 6$ are the aerodynamic friction and translational drag coefficients.

The system's inputs are defined as $u_{1}, u_{2}, u_{3}$ and $u_{4}$ that represent the total thrust force in the z-axis, the roll, pitch and yawing torques, respectively:

$$
\boldsymbol{u}=\left[\begin{array}{l}
u_{1} \\
u_{2} \\
u_{3} \\
u_{4}
\end{array}\right]=\left[\begin{array}{cccc}
b & b & b & b \\
0 & -l b & 0 & l b \\
-l b & 0 & l b & 0 \\
d & -d & d & -d
\end{array}\right]\left[\begin{array}{c}
\omega_{1}^{2} \\
\omega_{2}^{2} \\
\omega_{3}^{2} \\
\omega_{4}^{2}
\end{array}\right]
$$

where $b$ is the thrust coefficient, $d$ is the drag coefficient, and $\omega_{i}$ is the angular speed of the $i^{\text {th }}$ rotor.

Taking

$\boldsymbol{x}=(\phi, \dot{\phi}, \theta, \dot{\theta}, \psi, \dot{\psi}, x, \dot{x}, y, \dot{y}, z, \dot{z})^{T} \in \mathbf{R}^{12}$ as the state vector, the dynamical nonlinear model of the studied quadrotor is obtained as follows:

$$
\dot{\boldsymbol{x}}=f(\boldsymbol{x}, \boldsymbol{u})=\left\{\begin{array}{l}
\dot{x}_{1}=x_{2} \\
\dot{x}_{2}=a_{1} x_{4} x_{6}+a_{3} \bar{\Omega}_{r} x_{4}+a_{2} x_{2}^{2}+b_{1} u_{2} \\
\dot{x}_{3}=x_{4} \\
\dot{x}_{4}=a_{4} x_{2} x_{6}+a_{6} \bar{\Omega}_{r} x_{2}+a_{5} x_{4}^{2}+b_{2} u_{3} \\
\dot{x}_{5}=x_{6} \\
\dot{x}_{6}=a_{7} x_{2} x_{4}+a_{8} x_{6}^{2}+b_{3} u_{4} \\
\dot{x}_{7}=x_{8} \\
\dot{x}_{8}=a_{9} x_{8}+\frac{1}{m}(c \phi c \psi s \theta+s \phi s \psi) u_{1} \\
\dot{x}_{9}=x_{10} \\
\dot{x}_{10}=a_{10} x_{10}+\frac{1}{m}(c \phi s \theta s \psi-s \phi c \psi) u_{1} \\
\dot{x}_{11}=x_{12} \\
\dot{x}_{12}=a_{11} x_{12}+\frac{c \phi c \theta}{m} u_{1}-g
\end{array}\right.
$$

Where,

$a_{1}=\frac{I_{y}-I_{z}}{I_{x}}, a_{2}=-\frac{\kappa_{4}}{I_{x}}, a_{3}=-\frac{J_{r}}{I_{x}}, a_{4}=\frac{\left(I_{z}-I_{x}\right)}{I_{y}}$, $a_{5}=-\frac{\kappa_{5}}{I_{y}}, a_{6}=-\frac{J_{r}}{I_{y}}, a_{7}=\frac{\left(I_{x}-I_{y}\right)}{I_{z}}, a_{8}=-\frac{\kappa_{6}}{I_{z}}$, $a_{9}=-\frac{\kappa_{1}}{m}, a_{10}=-\frac{\kappa_{2}}{m}, a_{11}=-\frac{\kappa_{3}}{m}, b_{1}=\frac{l}{I_{x}}, b_{2}=\frac{l}{I_{y}}$ and $b_{3}=\frac{1}{I_{z}}$. 


\section{ADAPTIVE FUZZY GAINS-SCHEDULING OF INTEGRAL SLIDING MODE CONTROLLERS}

\section{A. Control Problem Statement}

The control aims to establish an adaptive controller that eliminates the attitude and altitude error dynamics of the quadrotor to assure high performances and robustness. The desired trajectories of the controlled states are defined as $\boldsymbol{x}_{d}=\left[\phi_{d}, \theta_{d}, \psi_{d}, z_{d}\right]^{T}$ and the actual ones are set as $\boldsymbol{x}=[\phi, \theta, \psi, z]^{T}$.

As shown in Fig. 2, the altitude controller takes an error signal $e$ as an input that introduce the gap between the desired altitude $z_{d}$ and the actual state $z$ and produces a control signal $u_{1}$. In a similar way, the attitude and heading controllers take as inputs the error signals between the desired roll $\phi_{d}$, pitch $\theta_{d}$ and yaw $\psi_{d}$ and their actual values $\phi, \theta$ and $\psi$ to produce the output control signals $u_{2}, u_{3}$ and $u_{4}$, respectively.

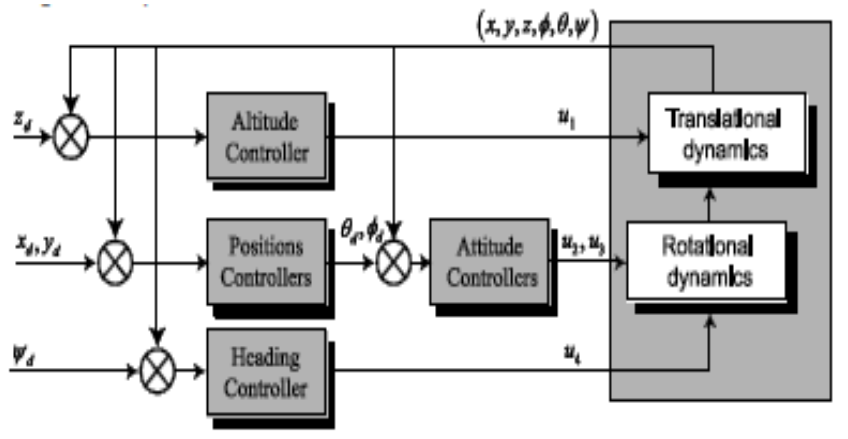

Fig. 2. Block diagram for altitude, attitude and heading controllers of the quadrotor.

The ISMC is a type of conventional SMC where an integral action is added to the general form of the sliding surface shape as proposed by [16]. The main aim is to lead the system states to the surface appropriately selected and conceive a stabilizing control law that maintains it. The sliding surface indicated by $S($.$) is specified as follows:$

$$
s_{i}(\boldsymbol{x}, t)=\left(\frac{d}{d t}+\lambda_{i}\right) e_{i}(\boldsymbol{x}, t)+\beta_{i} \int_{0}^{\infty} e_{i}(\boldsymbol{x}, t) d t
$$

where $\boldsymbol{x}$ denotes the accessible variables (states), $e_{i}(\boldsymbol{x}, t)$ is the tracking errors defined as $e_{i}(\boldsymbol{x}, t)=\boldsymbol{x}_{d}(t)-\boldsymbol{x}(t), \lambda_{i}$ is a positive constant that interprets the dynamics of the surface and $\beta_{i}$ is the integral gain, $i \in\{\phi, \theta, \psi, z\}$.

The first time derivative of the sliding surface of (7) is given by:

$$
\dot{s}(\boldsymbol{x}, t)=\ddot{e}(\boldsymbol{x}, t)+\lambda_{i} \dot{e}(\boldsymbol{x}, t)+\beta_{i} e(\boldsymbol{x}, t)
$$

As shown in [14], [19], [20], the sliding control law includes two terms as given by the following equation:

$$
u(t)=u_{e q}(t)+u_{D}(t)
$$

with $u_{e q}(t)$ indicate the equivalent control which defines the behavior of the system when the perfect sliding regime is settled, and $u_{D}(t)$ is a discontinuous function, called switching control, obtained by verifying of the condition of the attractiveness [1]. It is helpful to make up the uncertainties of the model and frequently is introduced as:

$$
u_{D}(t)=-K_{i} \operatorname{sgn}\left(s_{i}(\boldsymbol{x}, t)\right)
$$

Where, $K_{i}$ presents a positive control parameter and $\operatorname{sgn}($.$) denotes the mathematical signum function defined as:$

$$
\operatorname{sgn}(s)= \begin{cases}1, & s>0 \\ 0, & s=0 \\ -1, & s<0\end{cases}
$$

Lyapunov stability analysis is the most common approach to demonstrate and to assess the stable convergence property of nonlinear controllers. Here, direct Lyapunov stability approach is used to consider the stability property of the suggested integral sliding mode controller. It consists to make a positive scalar function, given by (12), for the system state variables and then chooses the control law that will decrease this function:

$$
\dot{V}(x, t)<0 \text { with } V(x, t)>0
$$

This Lyapunov function can be chosen, to prove the closedloop stability, as follows:

$$
V(t)=\frac{1}{2} s^{2}(t)
$$

Where, $V(0)=0$ and $V(t)>0$ for $s(t) \neq 0$.

The derivative of this above function is negative when the following expression, called the reaching condition [1], is checked:

$$
s(\boldsymbol{x}, t) \dot{s}(\boldsymbol{x}, t)<0
$$

For the quadrotor's altitude stabilization, we consider the following reduced model of such a flight dynamics:

$$
\ddot{z}=a_{11} \dot{z}+\frac{c \phi c \theta}{m} u_{1}-g
$$

The design issue is to force the performance of the system states to the desired trajectories which are known. While 
considering the reference trajectories $\dot{z}_{d}$ and $z_{d}$ which are the desired velocity and altitude, respectively, we define the relative tracking error by:

$$
e_{z}=z-z_{d}
$$

Referring to (8), the corresponding sliding surface is defined as follows:

$$
s_{z}(t)=\dot{e}_{z}(t)+\lambda_{z} e_{z}(t)+\beta_{z} \int_{0}^{\infty} e_{z}(t) d t
$$

Where, $\lambda_{z}>0$ and $\beta_{z}>0$ are the effective design parameters for the ISMC law.

According to (8) and (17), the integral sliding mode control law for the quadrotor's altitude dynamics is expressed as follows:

$$
u_{1}=\frac{m}{c \phi c \theta}\left[-a_{11} x_{12}-\lambda_{z} \dot{z}-\beta_{z}\left(z-z_{d}\right)-g\right]-K_{z} \operatorname{sgn}\left(s_{z}\right)
$$

Where, $K_{z}>0$ is a design ISMC parameter and $\phi, \theta \in]-\pi / 2, \pi / 2[$ to avoid singular positions.

So, while following the same steps as for the altitude dynamics, the integral sliding mode control laws $u_{2}, u_{3}$ and $u_{4}$ responsible of the roll, pitch and yaw dynamics stabilization, respectively, are calculated as follows:

$$
\begin{aligned}
& u_{2}=\frac{1}{b_{1}}\left[-a_{1} x_{4} x_{6}-a_{3} \bar{\Omega}_{r} x_{4}-a_{2} x_{2}^{2}-\lambda_{\phi} x_{2}-\beta_{\phi} e_{\phi}\right]-K_{\phi} \operatorname{sgn}\left(s_{\phi}\right) \\
& u_{3}=\frac{1}{b_{2}}\left[-a_{4} x_{2} x_{6}-a_{6} \bar{\Omega}_{r} x_{2}-a_{5} x_{4}^{2}-\lambda_{\theta} x_{4}-\beta_{\theta} e_{\theta}\right]-K_{\theta} \operatorname{sgn}\left(s_{\theta}\right) \\
& u_{4}=\frac{1}{b_{3}}\left[a_{7} x_{2} x_{4}+a_{8} x_{6}^{2}-\lambda_{\psi} x_{4}-\beta_{\psi} e_{\psi}\right]-K_{\psi} \operatorname{sgn}\left(s_{\psi}\right)
\end{aligned}
$$

Where, $\lambda_{i}>0, \quad \beta_{i}>0$ and $K_{i}>0$ are the effective design parameters for the ISMC-based stabilization of the roll, pitch and yaw motions, $i \in\{\phi, \theta, \psi\}$.

\section{B. Fuzzy Gains-Scheduling of Integral Sliding Mode Controllers}

The fuzzy gains scheduling scheme of Fig. 3 is proposed for the ISMC parameters selection and tuning. Such fuzzy inference mechanisms adjust with an adaptive manner all ISMC gains leading to a systematic selection approach for ISMC design. As depicted in Fig. 3, both gains of the sliding surfaces and sign functions shown in (19) to (21), will be generated using fuzzy supervisors FLS1 and FLS2 based on fuzzy rules and reasoning.

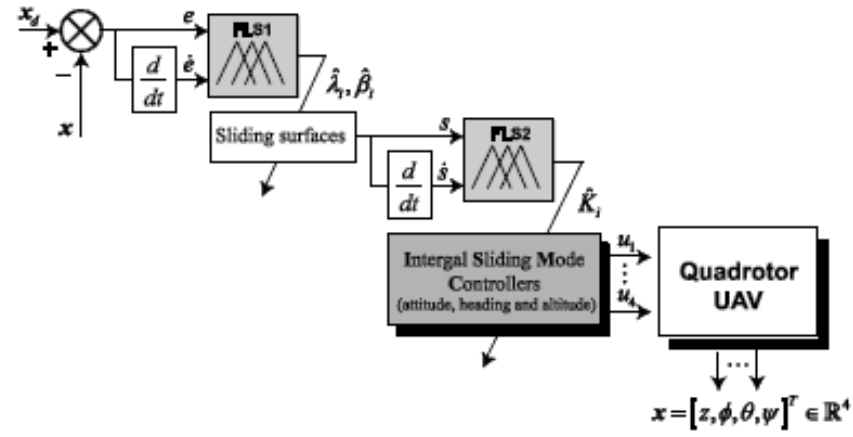

Fig. 3. Proposed fuzzy gains-scheduled integral sliding mode controllers.

The proposed fuzzy inference system FLS1 has two inputs $e(t)$ and its derivative $\dot{e}(t)$ and two outputs $\lambda_{i}$ and $\beta_{i}$ gains. The sliding surface gains are no longer fixed values. In fact, the gains are calculated at each sample period according to the evolution of the error. The decision-making outputs are obtained using a Max-Min fuzzy inference method where the crisp output is calculated by the center of gravity defuzzification technique. Tables I and II give the defined linguistic rules with the following assigned levels of the fuzzy inputs/outputs: N (Negative), NB (Negative Big), Z (Zero), P (Positive), PS (Positive Small), PM (Positive Medium), NB (Negative Big) and PB (Positive Big). All membership functions are defined with triangular and uniformly distributed shape.

TABLE. I. FuZZY RULES FOR $\lambda_{i}$ VARIABLES.

\begin{tabular}{|l|l|l|l|l|l|l|}
\hline \multicolumn{2}{|c|}{} & \multicolumn{5}{l}{$l$} \\
\cline { 3 - 7 } \multicolumn{2}{|c|}{} & $\mathrm{NB}$ & $\mathrm{N}$ & $\mathrm{Z}$ & $\mathrm{P}$ & $\mathrm{PB}$ \\
\hline \multirow{4}{*}{$\dot{e}$} & $\mathrm{NB}$ & $\mathrm{Z}$ & $\mathrm{Z}$ & $\mathrm{Z}$ & $\mathrm{PS}$ & $\mathrm{PM}$ \\
\cline { 2 - 7 } & $\mathrm{N}$ & $\mathrm{Z}$ & $\mathrm{PS}$ & $\mathrm{PS}$ & $\mathrm{PS}$ & $\mathrm{PM}$ \\
\cline { 2 - 7 } & $\mathrm{Z}$ & $\mathrm{Z}$ & $\mathrm{PS}$ & $\mathrm{PM}$ & $\mathrm{P}$ & $\mathrm{PB}$ \\
\cline { 2 - 7 } & $\mathrm{P}$ & $\mathrm{PM}$ & $\mathrm{P}$ & $\mathrm{P}$ & $\mathrm{P}$ & $\mathrm{PB}$ \\
\cline { 2 - 7 } & $\mathrm{PB}$ & $\mathrm{PM}$ & $\mathrm{P}$ & $\mathrm{PB}$ & $\mathrm{PB}$ & $\mathrm{PB}$ \\
\hline
\end{tabular}

TABLE. II. FuZZy RULES For $\beta_{i}$ VARIABLES.

\begin{tabular}{|l|l|l|l|l|l|l|}
\hline \multicolumn{2}{|c|}{} & \multicolumn{5}{l}{$l$} \\
\cline { 3 - 7 } \multicolumn{2}{|c|}{} & NB & N & Z & P & PB \\
\hline \multirow{4}{*}{$\dot{e}$} & NB & PB & Z & PB & PS & Z \\
\cline { 2 - 7 } & N & PB & P & P & PM & Z \\
\cline { 2 - 7 } & Z & P & P & PM & PS & Z \\
\cline { 2 - 7 } & P & PM & P & PS & PS & Z \\
\cline { 2 - 7 } & PB & PM & PS & Z & Z & Z \\
\hline
\end{tabular}

In this proposed supervision strategy, a set of linguistic rules in the form of Eq. (22) is used in the fuzzy inference block FLS1:

$$
\text { If } e_{i}(\boldsymbol{x}) \text { is } \boldsymbol{A}_{i} \text { and } \dot{e}_{i}(\boldsymbol{x}) \text { is } \boldsymbol{B}_{i} \text { then } \lambda_{i} \text { is } \boldsymbol{C}_{i} \text { and } \beta_{i} \text { is } \boldsymbol{D}_{i}
$$


where $\boldsymbol{A}_{i}, \boldsymbol{B}_{i}, \boldsymbol{C}_{i}$ and $\boldsymbol{D}_{i}$ are the fuzzy sets corresponding to $e_{i}(x), \dot{e}_{i}(x), \lambda_{i}$ and $\beta_{i}$ linguistic variables, respectively.

The switching gains $K_{i}$ are the main parameters to rise above perturbation and external interaction. Usually, the chattering amplitude of the controller is proportional to $K_{i}$, so the chattering could be diminished by setting this gain adaptively. The gains $K_{i}$ should be smaller when it is near to the sliding surface and larger when it is farther [16].

As illustrated in Fig. 3, $K_{i}$ gains will be generated using a second fuzzy supervisor, denoted as FLS2, where the sliding surface $s_{i}(\boldsymbol{x})$ and its differential $\dot{s}_{i}(\boldsymbol{x})$ are selected as inputs and $K_{i} i \in\{\phi, \theta, \psi, z\}$ are selected as outputs. The fuzzy rules for the proposed FLS2 are given in Table III.

TABLE. III. FuZZY RULES For $K_{i}$ VARIABLes

\begin{tabular}{|l|l|l|l|l|l|l|}
\hline \multicolumn{2}{|c|}{} & \multicolumn{3}{l}{$s$} \\
\cline { 3 - 7 } \multicolumn{2}{|c|}{} & Z & PS & PM & P & PB \\
\hline \multirow{4}{*}{$\dot{s}$} & Z & PB & PB & P & P & PM \\
\cline { 2 - 8 } & PS & PB & P & P & PM & PS \\
\cline { 2 - 7 } & PM & PB & P & PM & PS & Z \\
\cline { 2 - 7 } & P & P & PM & PS & PS & Z \\
\cline { 2 - 7 } & PB & PM & PS & PS & Z & Z \\
\hline
\end{tabular}

The decision-making outputs are obtained using a MaxMin fuzzy inference and the crisp output is calculated by the center of gravity defuzzification method. A set of linguistic rules in the form of (23) is used in such a fuzzy supervisor to determine the gains $K_{i}$ :

$$
\text { If } s_{i}(\boldsymbol{x}) \text { is } \boldsymbol{E}_{i} \text { and } \dot{s}_{i}(\boldsymbol{x}) \text { is } \boldsymbol{F}_{i} \text { then } K_{i} \text { is } \boldsymbol{G}_{i}
$$

where $\boldsymbol{E}_{i}, \boldsymbol{F}_{i}$ and $\boldsymbol{G}_{i}$ are the fuzzy sets corresponding to $s_{i}(\boldsymbol{x}), \dot{s}_{i}(\boldsymbol{x})$ and $K_{i}$ linguistic variables, respectively.

\section{NUMERICAL RESULTS AND DISCUSSIONS}

The simulations have been established to validate the proposed adaptive fuzzy gains-scheduled ISMC approach. The physical parameters of the quadrotor UAV are given in Table IV.

The initial states of the quadrotor are set as $[x, y, z]=[0,0,0]$ and $[\phi, \varphi, \psi]=[0,0,0]$ which means that the quadrotor is initially on the ground. The purpose of the designed adaptive fuzzy sliding mode controllers is to drive the rotorcraft to rise to 4 meters high and then keep hovering. At the same time, the quadrotor is controlled to bring the system states to be stabilized around the desired references $[0.9 ; 0.5$; $0.5] \mathrm{rad}$.
TABLE. IV. QUADROTOR'S MODEL PARAMETERS

\begin{tabular}{|l|l|l|}
\hline Symbol & Description & Value \\
\hline$b$ & Lift coefficient & $2.984 \times 10^{-5} N . s^{2} / \mathrm{rad}^{2}$ \\
\hline$d$ & Drag coefficient & $3.30 \times 10^{-7} N . s^{2} / \mathrm{rad}^{2}$ \\
\hline$m$ & Mass & $1.1 \mathrm{Kg}$ \\
\hline$l$ & Arm length & $0.50 \mathrm{~m}$ \\
\hline$J_{r}$ & Motor inertia & $2.8385 \times 10^{-5} \mathrm{~N} . \mathrm{m}^{-s^{-2}} / \mathrm{rad}$ \\
\hline$I$ & Quadrotor inertia & diag $(0.005,0.005,0.010)$ \\
\hline$g$ & $\begin{array}{l}\text { Acceleration of the } \\
\text { gravity }\end{array}$ & $9.81{\mathrm{~m} . \mathrm{s}^{-2}}$ \\
\hline
\end{tabular}

Unlike the conventional ISMC approach, the control gains $\lambda_{i}, \beta_{i}$ and $K_{i}$ of the proposed AFGS-ISMC are timevarying thanks to the supervisors FLS1 and FLS2 using the fuzzy rules of Tables I, II and III. The obtained fuzzy surfaces for all $\lambda_{i}, \beta_{i}$ and $K_{i}$ decision parameters are given in Fig. 4, 5 and 6 , respectively.

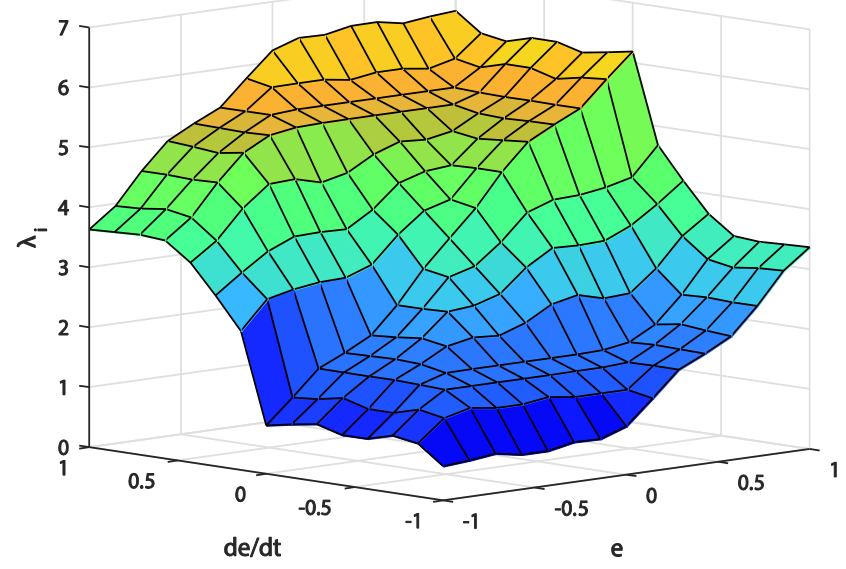

Fig. 4. Fuzzy surface for the $\lambda_{i}$ gains.

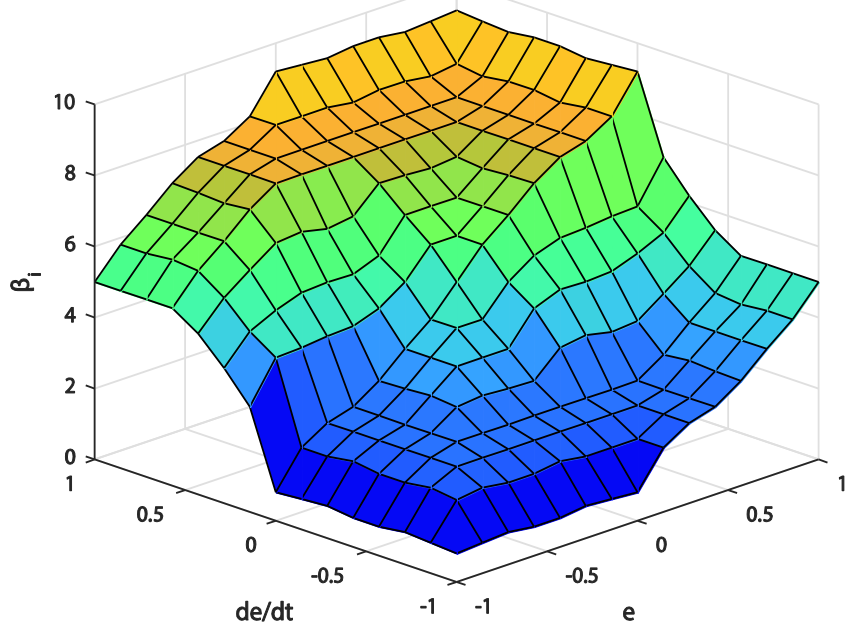

Fig. 5. Fuzzy surface for the $\beta_{i}$ gains. 


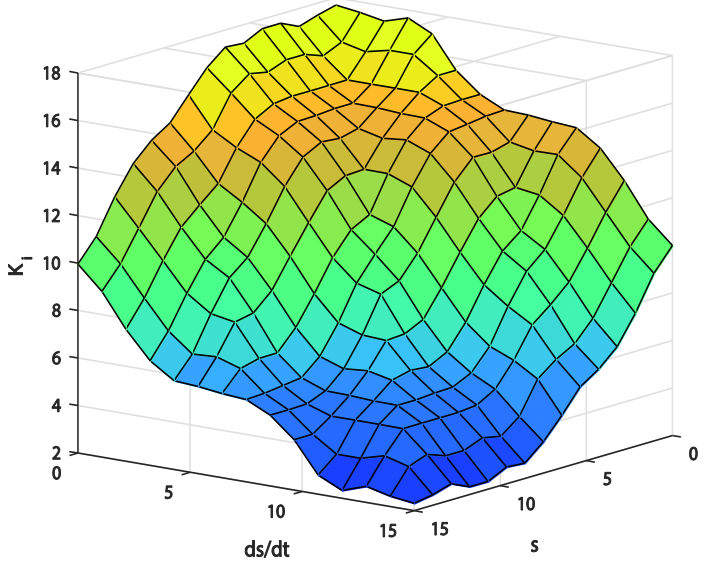

Fig. 6. Fuzzy surface for the $K_{i}$ gains.

In this simulation scenario, external disturbances are applied on the quadrotor's outputs at the simulation time $t=10 \mathrm{sec}$. The generated AFGS-based ISMC gains for the closed-loop altitude and attitude dynamics are shown in Fig. 7 to Fig. 10. All these controller's gains become time-varying which are more adapted and efficient to uncertainties and disturbances rejection as well as for the unwanted chattering phenomenon's attenuation.

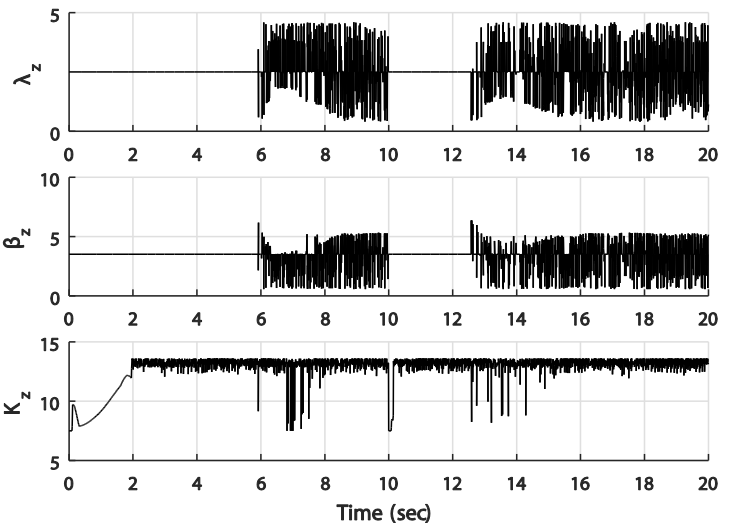

Fig. 7. Time evolution of the fuzzy gains-scheduled integral sliding mode gains: altitude dynamics.
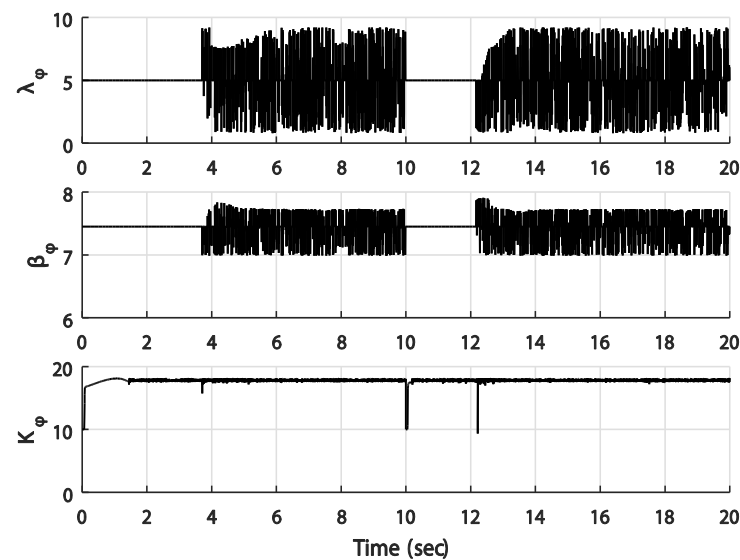

Fig. 8. Time evolution of the fuzzy gains-scheduled integral sliding mode gains: roll dynamics.
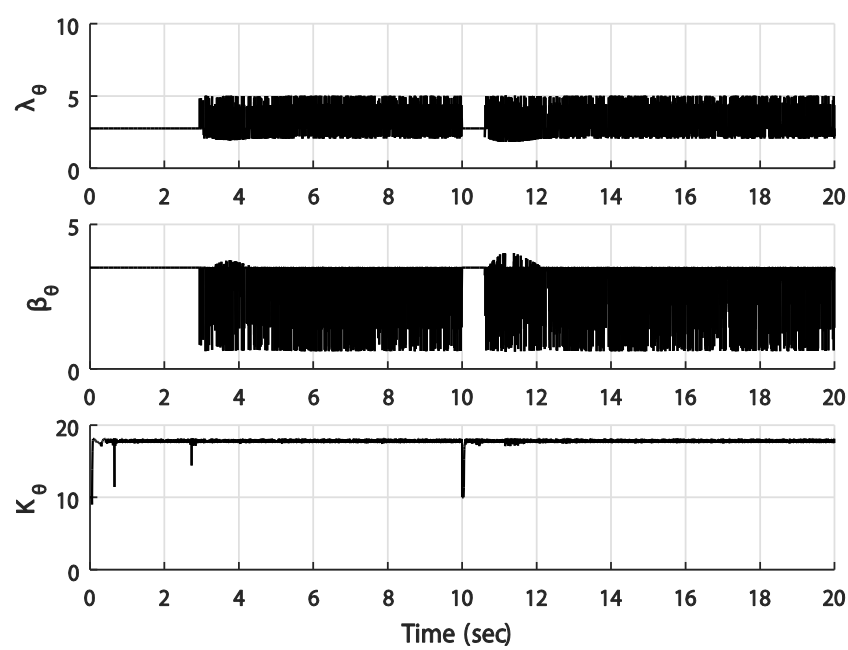

Fig. 9. Time evolution of the fuzzy gains-scheduled integral sliding mode gains: pitch dynamics.
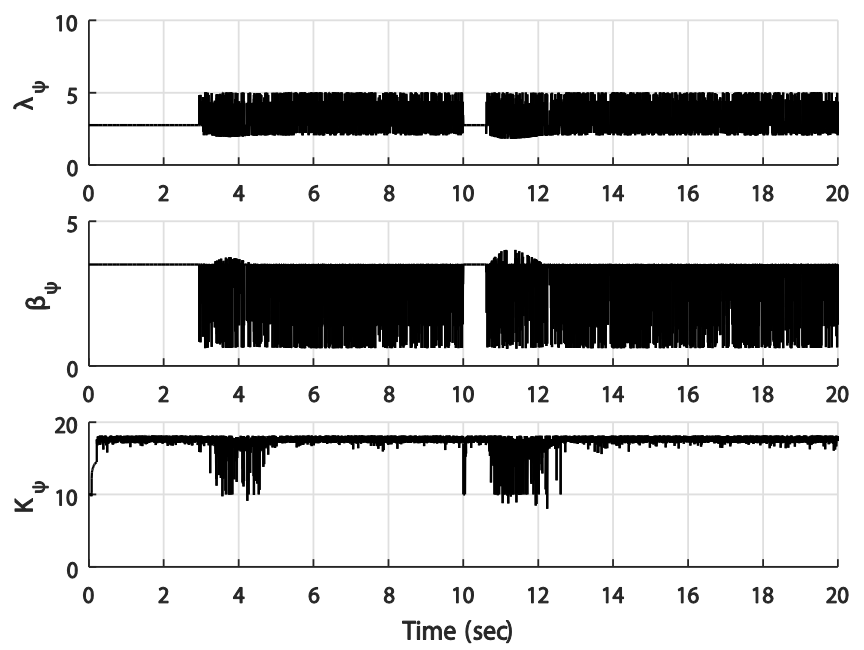

Fig. 10. Time evolution of the fuzzy gains-scheduled integral sliding mode gains: yaw dynamics.

So as to show the time-domain performances of the proposed AFGS-based sliding mode controllers, a comparison to the conventional ISMC case, using both $\operatorname{sgn}($.$) and$ sat (.) based sliding functions, has been carried out and relative results are pictured in Fig. 11 to 14 for the altitude, roll, pitch and yaw motions, respectively. From these closedloop step responses, it is verified that both ISMC and AFGSISMC strategies are effective for the attitude and altitude control. The AFGS-based controllers provide better results in terms of disturbances rejection and transient response damping. A slight overshoot is observed in the AFGS-based controllers' responses but with the advantage of high performance tracking responses. The steady-state precision and fastness of the AFGS-based controlled UAV dynamics are more improved related to the standard ISMC approach. Indeed, the dynamic response of the ISMC is delayed and the steady-state regime is reached within 3 seconds when applying an external disturbances. 


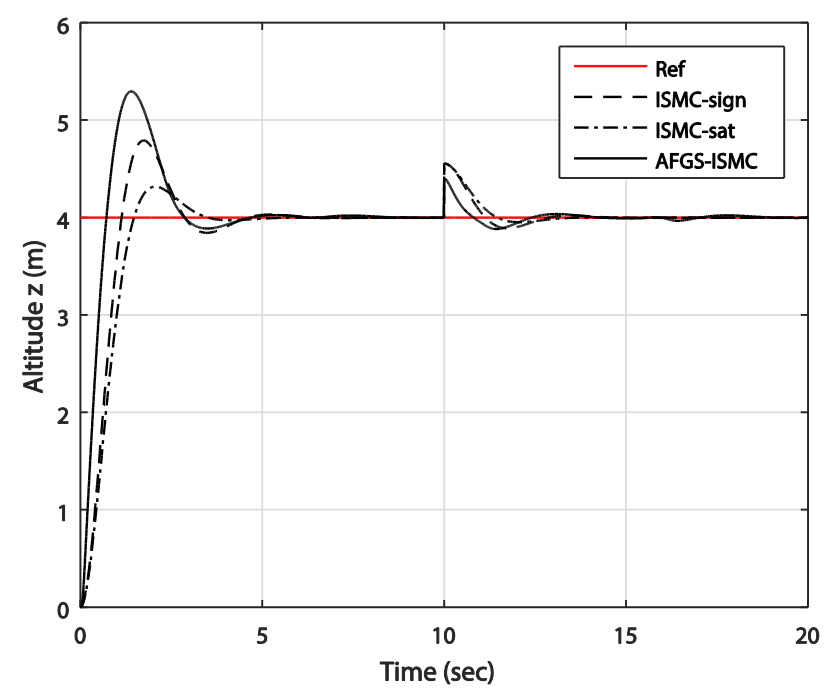

Fig. 11. Closed-loop step responses of the altitude dynamics.

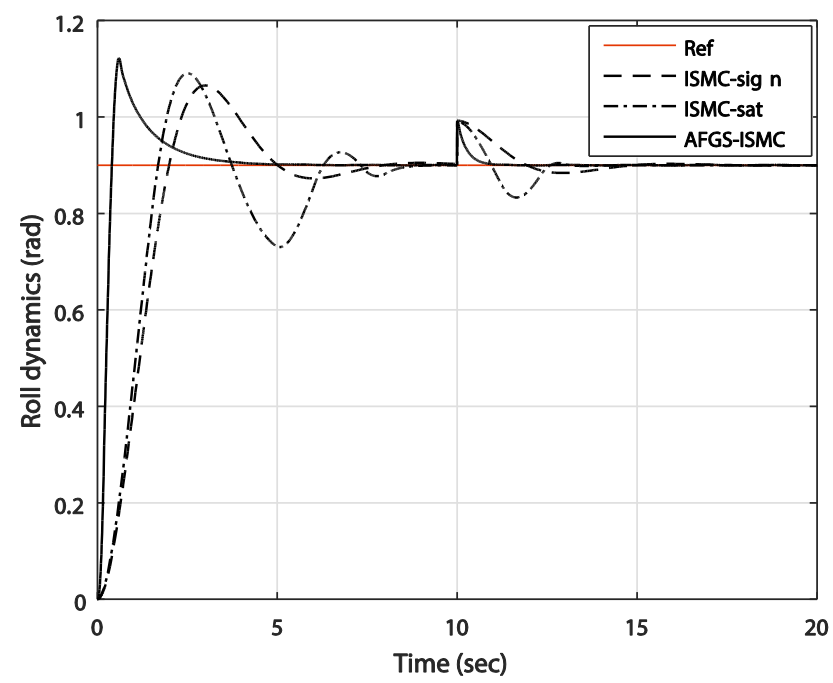

Fig. 12. Closed-loop step responses of the roll dynamics.

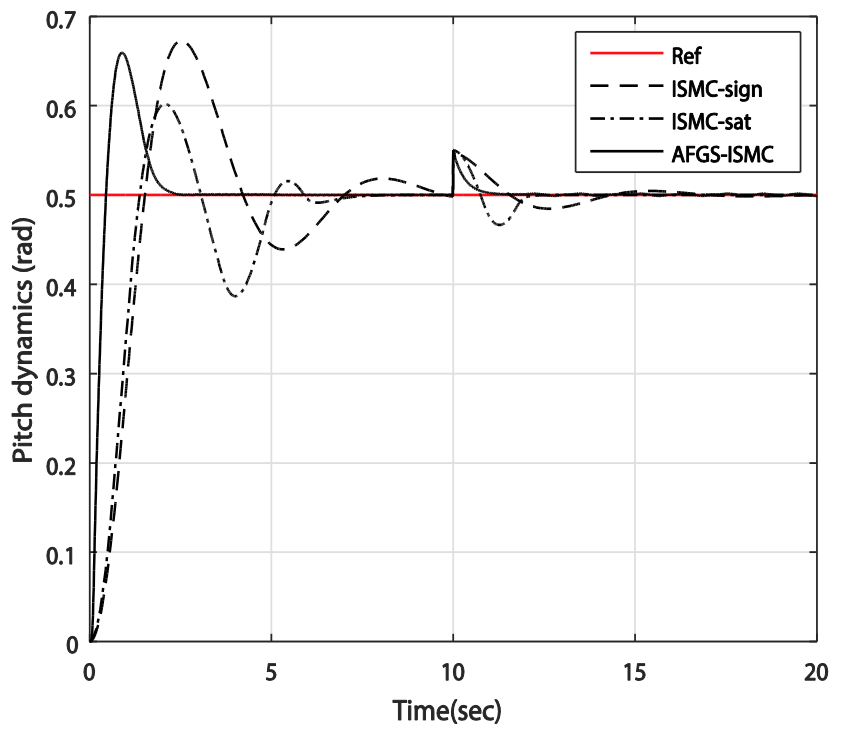

Fig. 13. Closed-loop step responses of the pitch dynamics.

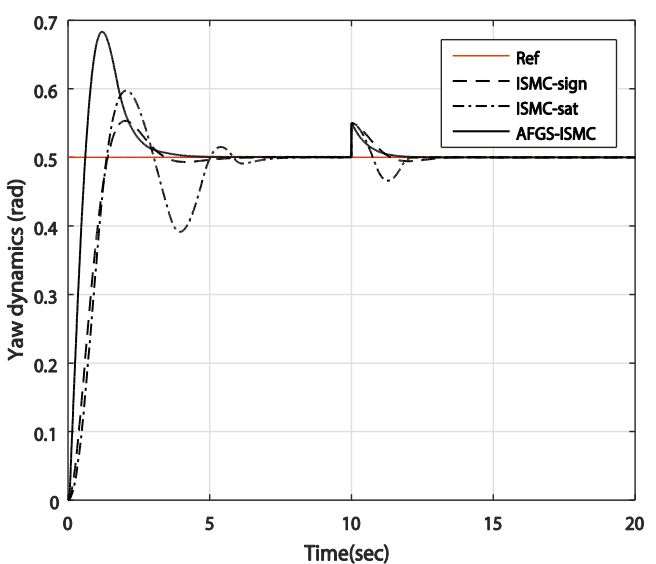

Fig. 14. Time-domain performances of the controlled yaw dynamics.

On the other hand, Fig. 15, 16, 17 and 18 display all control laws for the quadrotor's dynamics stabilization. The chattering phenomena are further reduced and the control laws are smoother due to the use of adaptive fuzzy gains-scheduling supervisors in the contrast to the classical ISMC without gains supervision mechanisms. Based on these results, the proposed free-chattering AFGS-based ISMC approach is promising in the definitive real-world implementation and hardware prototyping of the designed flight controllers for such a type of VTOL vehicles.

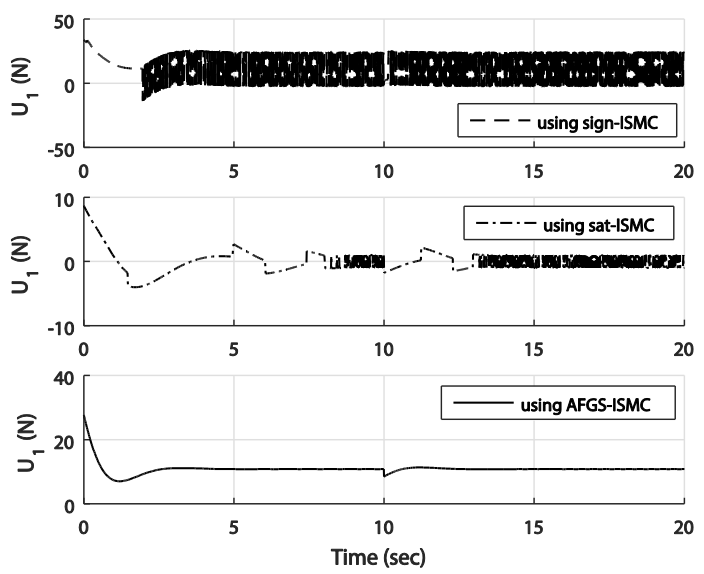

Fig. 15. Control laws for the altitude dynamics stabilization.
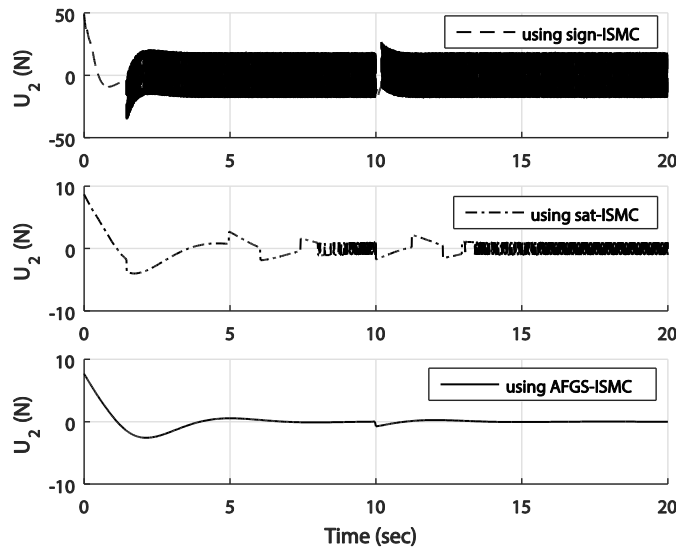

Fig. 16. Control laws for the roll dynamics stabilization. 


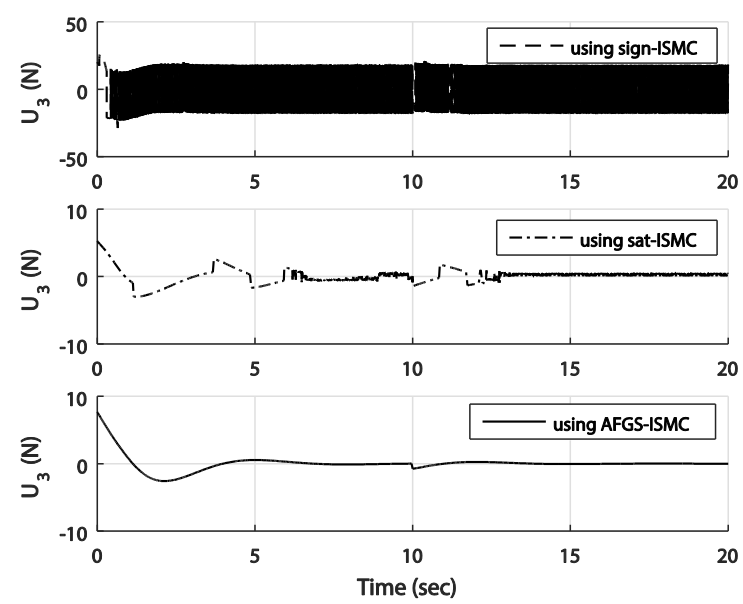

Fig. 17. Control laws for the pitch dynamics stabilization.
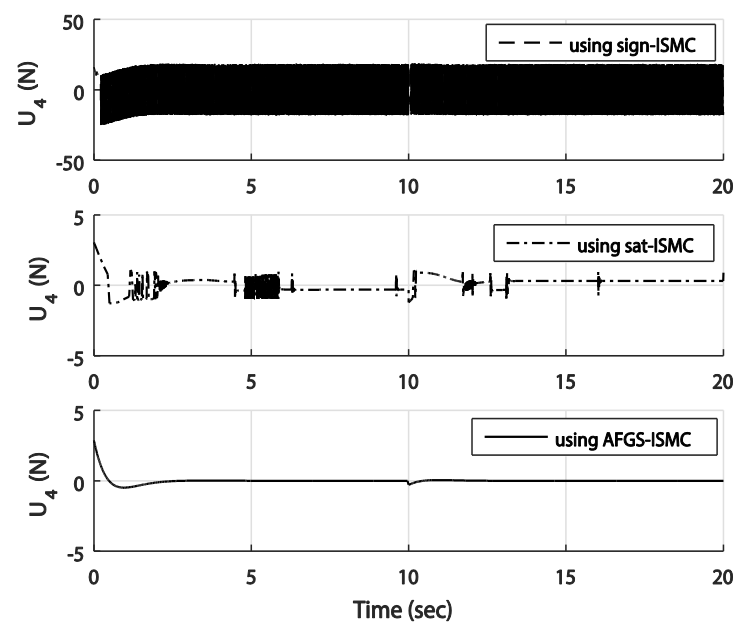

Fig. 18. Control laws for the yaw dynamics stabilization.

\section{CONCLUSION}

In this work, an adaptive fuzzy gains-scheduled integral sliding mode control approach is suggested and successfully applied for a quadrotor UAV. With a remarkable decreasing of the chattering phenomenon, this strategy is proposed to address the stabilization problem of the attitude and altitude dynamics of the studied vehicle. The dynamical model of the quadrotor was firstly settled using the Newton-Euler formalism. Then, the design of adaptive fuzzy gains-scheduled integral sliding mode controllers is detailed for each flight dynamics. In order to select and tune the gains of sliding controllers, as effective decision parameters, two fuzzy logic supervisors are proposed and implemented to make the controllers' gains varying adaptively. A comparison with the conventional ISMC strategy was made in terms of time-domain performances and chattering phenomenon attenuation. Through the simulation results, the proposed fuzzy gains-scheduling approach outperforms all other classical ISMC techniques with sign and saturation-based sliding functions. The design of integral sliding mode controllers with time-varying gains further enhances high closed-loop performances of the rotorcraft UAV in terms of stability and robustness. Forthcoming works deal with the Hardware-In-the-Loop (HIL) co-simulation of the designed ISMC approach.

\section{REFERENCES}

[1] S.Vaidyanathan and C-H.LIEN,(Ed.), "Applications of Sliding Mode Control in Science and Engineering “,Springer-Verlag, Germany ,2017.

[2] R. Lozano, (Ed.), "Unmanned Aerial Vehicles: Embedded Control", ISTE and John Wiley \& Sons, London UK and Hoboken USA,2013.

[3] K.. Nonami, F. Kendoul, S. Suzuki, W. Wang, and D. Nakazawa, "Autonomous Flying Robots: Unmanned Aerial Vehicles and Micro Aerial Vehicles", Springer, New York,2010.

[4] N. Ben Ammar, S. Bouallègue, J. Haggège, and S. Vaidyanathan "Chattering Free Sliding Mode Controller Design for a Quadrotor Unmanned Aerial Vehicle", In S. Vaidyanathan and C-H. Lien (Eds.), Applications of Sliding Mode Control in Science and Engineering, Springer-Verlag, pp. 61-79, Germany,2017.

[5] N. Ben Ammar, S. Bouallègue and J. Haggège. "Modeling and Sliding Mode Control of a Quadrotor Unmanned Aerial Vehicle", In Proceedings of the $3^{\text {th }}$ International Conference on Automation, Control Engineering and Computer Science, pp. 834-840, Hammamet, Tunisia,2016.

[6] I. Fantoni, and R. Lozano, "Nonlinear Control for Underactuated Mechanical Systems", Springer-Verlag, London, 2012.

[7] J. Guerrero, and R. Lozano, "Flight Formation Control", Wiley-ISTE, UK, 2012.

[8] M.O. Efe, "Robust Low Altitude Behavior Control of a Quadrotor Rotorcraft through Sliding Modes", In Proceedings of the Mediterranean Conference on Control and Automation, pp.1-6, Athens, Greece ,2007.

[9] H.K . Khalil, "Nonlinear Systems", Prentice Hall, New Jersey, 2002.

[10] B.M . Meghni, D. Dib, A.T. Azar, "A Second-order sliding mode and fuzzy logic control to Optimal Energy Management in PMSG Wind Turbine with Battery Storage", Neural Computing and Applications, Springer. DOI 10.1007/s00521-015-2161-z, 2016.

[11] H. Mekki, D. Boukhetala, and A.T. Azar, "Sliding Modes for Fault Tolerant Control", In: A.T Azar, Q. Zhu, (eds.), Advances and Applications in Sliding Mode Control systems, Studies in Computational Intelligence book Series, vol. 576, pp. 407-433, Springer-Verlag GmbH Berlin/Heidelberg. DOI 10.1007/978-3-319-11173-5_15, 2015.

[12] X. Li and J. Zhou, "A sliding mode control design for mismatched uncertain systems based on states transformation scheme and chattering alleviating scheme", Transactions of the Institute of Measurement and Control, DOI:0142331216680351, 2017.

[13] Y. Liu and Y. Niu "Sliding mode control for uncertain switched systems subject to state and input delays", Transactions of the Institute of Measurement and Control, DOI: 0142331217724195 , 2017.

[14] B.B. Musmade and B.M. Patre, "Sliding mode control design for robust regulation of time-delay processes", Transactions of the Institute of Measurement and Control, vol. 37, no. 6, pp. 699-707, 2015.

[15] I. González-Hernández, S. Salazar, A. E. Rodríguez-Mata, F. MuñozPalacios, R. López, and R. Lozano, "Enhanced Robust Altitude Controller via Integral Sliding Modes Approach for a Quad-Rotor Aircraft: Simulations and Real-Time Results," J. Intell. Robot. Syst. Theory Appl., vol. 88, no. 2-4, pp. 313-327, 2017.

[16] J-J.E. Slotine and W. Li, "Applied Nonlinear Control”, Prentice-Hall, Englewood Cliffs, New Jersey, 1991.

[17] J-Z. Zhang and H-T. Zhang,"Vehicle Stability Sliding Mode Control Based on Fuzzy Switching Gain Scheduling", In International Conference on Measuring Technology and Mechatronics Automation ,pp. 1067-1070, Changsha City, China,2010.

[18] H. Sira-Ramirez, "On the dynamical sliding mode control of nonlinear systems", International Journal of Control, vol. 5, no. 57, pp.1039$1061,1993$.

[19] Y. Shtessel, C. Edwards, L. Fridman and A. Levant, "Sliding mode control and observation", Springer, New York , 2014.

[20] V. Utkin, J. Guldner and J. Shi, "Sliding mode control in electromechanical systems", CRC Press, 2009.

[21] S. Bouabdallah, A. Noth and R. Siegwart, "PID vs. LQ Control Techniques Applied to an Indoor Micro Quadrotor", In Proceedings of the IEEE/RSJ International Conference on Intelligent Robots and Systems, pp. 2451-2456, Sendai, Japan , 2004. 
[22] F. Castaños and L. Fridman, "Analysis and design of integral sliding manifolds for systems with unmatched perturbations", IEEE Transactions on Automatic Control, vol. 51, no. 5, pp.853-858, 2006.

[23] V. Utkin and J. Shi, "Integral sliding mode in systems operating under uncertainty conditions", In Proceedings of the $35^{\text {th }}$ IEEE Conference on Decision Control, pp. 4591-4596, Dec, Kobe, 1996.

[24] K. P. Ramesh, S. Kamal, A. Chalanga, and B. Bandyopadhyay, "Multivariable continuous integral sliding mode control," in International Workshop on Recent Advances in Sliding Modes, RASM 2015.

[25] M. Rubagotti, D.M. Raimondo, A. Ferrara and L. Magni, "Robust model predictive control with integral sliding mode in continuous time ampled-data nonlinear systems", IEEE Transactions on Automatic Control, vol. 56, no. 3, pp. 556-570, 2011.

[26] K. Nonaka and H. Sugizaki, "Integral Sliding Mode Altitude Control for a Small Model Helicopter with Ground Effect Compensation", In Proceedings of the 2011 American Control Conference, pp. 202-207, San Francisco, USA, 2011.

[27] R.N.T. Dief and G.M. Abdelhady, "Attitude and altitude stabilization of quad rotor using parameter estimation and self-tuning controller", In Proceedings of the AIAA Atmospheric Flight Mechanics Conference, Dallas , 2015.
[28] S.M. Mallikarjunan, B. Nesbitt and E. Kharisov, "L1 adaptive controller for attitude control of multi-rotors", In Proceedings of the AIAA Guidance, Navigation, and Control Conference, Minneapolis, Minnesota, 2012.

[29] S.Bouallègue and R.Fessi, "Rapid control prototyping and PIL cosimulation of a quadrotor UAV based on NI myRIO-1900 board" International Journal of Advanced Computer Science and Applications, vol.7, no.6, pp.26-35, 2016.

[30] W. Siler, and J.J. Buckley, "Fuzzy expert systems and fuzzy reasoning", John Wiley \& Sons, New Jersey, 2005.

[31] E. Mohammadi and M. Montazeri-Gh, "A fuzzy-based gas turbine fault detection and identification system for full and part-load performance deterioration", Aerospace Science and Technology, vol. 46, pp. 82-93, 2015.

[32] S., Yin, P. Shi, and H.Y. Yang, "Adaptive fuzzy control of strictfeedback nonlinear time-delay systems with unmodeled dynamics", IEEE Transactions on Cybernetics, vol. 46, no. 8, pp. 1-12,2015.

[33] I. Eker, and S.A. Akinal, "Sliding mode control with integral augmented sliding surface: design and experimental application to an electromechanical system", Journal of Electrical Engineering, vol. 97, no. 3, pp. 90-189,2008. 